\title{
Analytical and Numerical Results on Global Dynamics of the Generalized Boussinesq Equation with Cubic Nonlinearity and External Excitation
}

\author{
Mingyuan Li, ${ }^{1,2,3,4}$ Wei Zhang $\mathbb{D}^{1,2}$ and Qiliang $W u \mathbb{i}^{5,6}$ \\ ${ }^{1}$ Beijing Key Laboratory of Nonlinear Vibrations and Strength of Mechanical Structures, Beijing University of Technology, \\ Beijing 100124, China \\ ${ }^{2}$ College of Mechanical Engineering, Beijing University of Technology, Beijing 100124, China \\ ${ }^{3}$ Big Data Applied Research and Collaborative Innovation Center, Inner Mongolia University of Finance and Economics, \\ Hohhot 010070, Inner Mongolia, China \\ ${ }^{4}$ School of Statistics and Mathematics, Inner Mongolia University of Finance and Economics, Hohhot 010070, \\ Inner Mongolia, China \\ ${ }^{5}$ School of Artificial Intelligence, Tiangong University, Tianjin 300387, China \\ ${ }^{6}$ Tianjin Key Laboratory of Advanced Technology of Electrical Engineering and Energy, Tiangong University, \\ Tianjin 300387, China
}

Correspondence should be addressed to Wei Zhang; sandyzhang0@yahoo.com and Qiliang Wu; qiliang_wu@hotmail.com

Received 13 November 2020; Revised 5 March 2021; Accepted 22 May 2021; Published 1 June 2021

Academic Editor: Diego Oliva

Copyright (C) 2021 Mingyuan Li et al. This is an open access article distributed under the Creative Commons Attribution License, which permits unrestricted use, distribution, and reproduction in any medium, provided the original work is properly cited.

This paper analytically and numerically presents global dynamics of the generalized Boussinesq equation (GBE) with cubic nonlinearity and harmonic excitation. The effect of the damping coefficient on the dynamical responses of the generalized Boussinesq equation is clearly revealed. Using the reductive perturbation method, an equivalent wave equation is then derived from the complex nonlinear equation of the GBE. The persistent homoclinic orbit for the perturbed equation is located through the first and second measurements, and the breaking of the homoclinic structure will generate chaos in a Smale horseshoe sense for the GBE. Numerical examples are used to test the validity of the theoretical prediction. Both theoretical prediction and numerical simulations demonstrate the homoclinic chaos for the GBE.

\section{Introduction}

The field of global dynamics for infinite-dimensional systems is becoming increasingly attractive for interdisciplinary research. This paper aims to develop a theoretical analysis method of global dynamics for infinite-dimensional dynamical systems. An analytical method is conducted to detect the chaotic waves and nonlinear dynamical behaviors of the generalized Boussinesq equation (GBE) with cubic nonlinearity and external excitation.

As a typical model in physics and mechanics, the Boussinesq equation (BE) possesses both dispersive and nonlinear attributes [1]. BE is a powerful tool to predict coastal hydrodynamics such as wave propagation, wave- current interaction, wave breaking, and nearshore circulation [2], even in extreme weather conditions such as typhoons and windstorms [3]. Many scholars have theoretically analyzed the characteristics of the BE. Weiss and his coworkers [4-6] explored Lax pairs, Bäcklund transformation, and the Painlevé property of the BE. The homogeneous balance method was extended to study the solution of traveling waves for the Boussinesq-Burgers equation [7]. Yang et al. [8] analyzed the global attractors and asymptotic behaviors for Schrödinger-Boussinesq equations. Based on the Hamiltonian structure, second- and fourth-order energy-preserving wavelet collocation schemes were proposed by Helal et al. [9] for the nonlinear coupled Schrödinger-Boussinesq model. Awais and Ibrahim [10] 
carried out studies on water waves in a fourth-order Boussinesq nonlinear model and analyzed its stability for soliton solutions. Considering the impact of diabatic force, the instability of Boussinesq equations was investigated [11]. In [12], a numerical analysis was conducted to investigate the sensitivities of coastal wave attenuation resulting from incident wave height, period, water depth, and vegetation configurations in a nonlinear model of Boussinesq equations. Wang and $\mathrm{Su}$ [13] studied the finite time blow-up and solvability of solutions in all space dimensions for the Cauchy problem of a Boussinesq equation with dissipation. Yildirim analyzed, theoretically and numerically, the boundary control problem [14] and active control [15] for the nonlinear Boussinesq system.

Traditionally, infinite-dimensional systems are described as PDEs (partial differential equations), and then the PDEs are truncated into ODEs (ordinary differential equations) [16-22]. Theoretically, ODEs are topologically equivalent to PDEs, but the dynamical phenomena cannot be perfectly exhibited. Hence, how to improve an analytical method of global dynamics for infinite-dimensional systems must be resolved still by scientists and scholars. In recent decades, a level of improvement in this area has been achieved. The first studies of the global perturbation technique for nonlinear PDEs were conducted by Li et al. [23, 24], Zeng [25], and Shatah and Zeng [26]. The persistent homoclinic orbits of nonlinear Schrödinger (NLS) and sine-Gordon (SG) equations were constructed by using invariant manifolds, foliations, and Melnikov analysis. Furthermore, Zhang et al. [27], Wu and Qi [28, 29], and Li et al. [30] improved the analytic theory for infinite-dimensional systems [23-26] to obtain the chaotic threshold of mechanical systems such as truss core sandwich plates, pipes conveying fluid, axial moving beams, and carbon nanotubes. The aforementioned literature focused on solid structure systems; however, analysis on global dynamics for fluid systems, e.g., GBE, remains lacking.

The objective of this study is to derive the analytical chaotic threshold of the GBE and indicate how the damping coefficient can clearly affect the dynamical responses of the GBE. The reductive perturbation method (RPM) $[27,30]$ is employed to transform the complicated PDE of the GBE into a topologically equivalent wave equation. The first and second measurements are then applied to explore the homoclinic chaos and the chaotic threshold for the wave equation. Afterward, numerical examples are also carried out using the differential quadrature method [27-29]. The simulating results confirm the validity of the theoretical prediction and illustrate larger damping coefficient resulting in the more complicated dynamical behaviors of the GBE.

\section{Motion Equation and Perturbation Analysis}

Boussinesq [31] assumed that the horizontal velocity is constant along the direction of water depth, and the vertical velocity is linearly distributed, obtaining the one-dimensional nonlinear water wave governing equation. Based on literature [31], the nonlinear governing equation of the cubic nonlinearity and harmonic excited GBE can be proposed as

$$
q_{t t}+\mu q_{t}-q_{x x}+\left(q^{3}+q_{x x}\right)_{x x}=\alpha \cos (\omega t),
$$

with the periodic boundary conditions

$$
\begin{aligned}
q(t) & =q(t+2 \pi), \\
q_{x}(t) & =q_{x}(t+2 \pi),
\end{aligned}
$$

where $\mu, \alpha$, and $\omega$ stand for the damping coefficient, amplitude, and frequency of the external force, respectively.

To carry out the analysis on the complicated dynamical responses of equation (1), the RPM is applied to simplify the formula, and the following formal solution of equation (1) yields:

$$
\begin{aligned}
q(x, t, \varepsilon) & =\varepsilon u(X, T) e^{i \phi}+c . c ., \\
\phi & =k x-\omega t
\end{aligned}
$$

where $X=\varepsilon(x-c t)$ and $T=\varepsilon^{2} t$.

Thus, the derivatives used in the RPM are given as

$$
\begin{aligned}
\frac{\partial}{\partial t} & \longrightarrow \frac{\partial}{\partial t}-\varepsilon^{1 / 2} c \frac{\partial}{\partial X}+\varepsilon \frac{\partial}{\partial T}, \\
\frac{\partial^{2}}{\partial t^{2}} & \longrightarrow \frac{\partial^{2}}{\partial t^{2}}-2 \varepsilon^{1 / 2} c \frac{\partial^{2}}{\partial X \partial t}+\varepsilon c^{2} \frac{\partial^{2}}{\partial X^{2}}+2 \varepsilon \frac{\partial^{2}}{\partial t \partial T}-2 \varepsilon^{3 / 2} c \frac{\partial^{2}}{\partial X \partial T}+\varepsilon^{2} \frac{\partial^{2}}{\partial T^{2}} \\
\frac{\partial}{\partial x} & \longrightarrow \frac{\partial}{\partial x}+\varepsilon^{1 / 2} \frac{\partial}{\partial X}, \\
\frac{\partial^{2}}{\partial x^{2}} & \longrightarrow \frac{\partial^{2}}{\partial x^{2}}+2 \varepsilon^{1 / 2} \frac{\partial^{2}}{\partial x \partial X}+\varepsilon \frac{\partial^{2}}{\partial X^{2}}, \\
\frac{\partial^{4}}{\partial x^{4}} & \longrightarrow \frac{\partial^{4}}{\partial x^{4}}+4 \varepsilon^{1 / 2} \frac{\partial^{4}}{\partial x^{3} \partial X}+6 \varepsilon \frac{\partial^{4}}{\partial x^{2} \partial X^{2}}+4 \varepsilon^{3 / 2} \frac{\partial^{4}}{\partial x \partial X^{3}}+\varepsilon^{2} \frac{\partial^{4}}{\partial X^{4}}
\end{aligned}
$$


Moreover, the following scale transformation is introduced:

$$
\mu \longrightarrow \varepsilon^{2} \mu
$$

By substituting equations (3)-(5) into equation (1) and removing the secular terms in the power of $\varepsilon$, the results are yielded as follows:

$O(\varepsilon):$

$$
\left(\omega^{2}-k^{2}-k^{4}\right) u=0
$$

$O\left(\varepsilon^{2}\right):$

$$
\left(c \omega-k+4 k^{3}\right) \frac{\partial u}{\partial X}=0
$$

$O\left(\varepsilon^{3}\right)$

$$
-2 i \omega \frac{\partial u}{\partial T}+\left(c^{2}+1-k^{2}\right) \frac{\partial^{2} u}{\partial X^{2}}-3 k^{2}|u|^{2} u-i \mu \omega u=0 .
$$

$$
\begin{aligned}
& O\left(\varepsilon^{4}\right): \\
& 2 c \frac{\partial^{2} u}{\partial X \partial T}+4 i k \frac{\partial^{3} u}{\partial X^{3}}-6 i k \frac{\partial|u|^{2} u}{\partial X}-\mu c \frac{\partial u}{\partial X}=0 .
\end{aligned}
$$

In order to derive the perturbed equation possessing the same form in $[23,24]$, equation (9) is integrated with respect to $X$, and the integral constant is $-2 i c \alpha e^{-i \beta \omega^{2} T}$. Letting $u \longrightarrow u e^{-i \beta \omega^{2} T}$, equation (9) is transformed into

$$
i \frac{\partial u}{\partial T}=\frac{\partial^{2} u}{\partial X^{2}}+\beta\left(|u|^{2}-\omega^{2}\right) u+i \frac{\mu}{2} u-i \alpha,
$$

where $X^{*}=\sqrt{(2 k / c)} X, \beta=3 k / c$, and the asterisk is dropped for convenience.

Hence, wave equation (10) has the same form in $[23,24]$ and is thus topologically equivalent to equation (1). Therefore, by using equation (10), the nonlinear characteristics of the wave equation for the GBE can be explored.

\section{Global Analysis}

The dynamical characteristics of equation (10) are further studied in this section. A bookkeeping parameter $\varepsilon$ is inserted and obtains the following equation:

$$
i \frac{\partial u}{\partial T}=\frac{\partial^{2} u}{\partial X^{2}}+\beta\left(|u|^{2}-\omega^{2}\right) u+i \varepsilon\left(\frac{\mu}{2} u-\alpha\right) .
$$

Equation (11) contains the invariant subspace

$$
\prod=\left\{u(X, T) \mid \partial_{X} u \equiv 0\right\},
$$

which owns the resonant torus:

$$
S=\left\{u \in \prod|u| \equiv \omega\right\} .
$$

When $\varepsilon=0$, we have

$$
i \frac{\partial u}{\partial T}=\frac{\partial^{2} u}{\partial X^{2}}+\beta\left(|u|^{2}-\omega^{2}\right) u
$$

with Hamiltonian

$$
H(u)=\int_{0}^{1}\left(\left|\partial_{X} u\right|^{2}+\beta \omega^{2}|u|^{2}-\frac{\beta}{2}|u|^{4}\right) \mathrm{d} X .
$$

Assuming $\beta>0$, it is observed that equation (14) is completely integrable and has a periodic solution [23-26]

$$
u_{0}(T)=r \exp \left[-i \beta\left(r^{2}-\omega^{2}\right) T+i T_{0}\right]
$$

as well as an analytical solution [23-26]

$$
u_{h}^{ \pm}(X, T)=\frac{\cos 2 p \cosh \bar{T}-i \sin 2 p \sinh \bar{T} \pm \sin p \cos X}{\cosh \bar{T} \mp \sin p \cos X} u_{0}(T),
$$

where

$$
\begin{aligned}
& \bar{T}=\phi\left(T+T_{1}\right), \\
& \phi=\sqrt{2 \beta r^{2}-\omega^{2}}, \\
& p=\arctan \sigma .
\end{aligned}
$$

The symbol \pm represents different parts of solutions $u_{h}^{ \pm}(X, T)$ being homoclinic to $u_{0}(T)$. Meanwhile, $u_{h}^{ \pm}(X, T)$ can also be seen as a heteroclinic orbit connecting different points in periodic orbit $u_{0}(T)$ due to the phase shifts, as presented in Figure 1. Figure 2 depicts the heteroclinic structure $u_{h}^{ \pm}(X, T)$ in space $(X, T,|u|)$, respectively. To analyze the dynamic characteristics of equation (11) near $S$, the following transformations are introduced:

$$
\begin{aligned}
u & =\sqrt{\omega^{2}+\sqrt{\varepsilon} I} \exp (i \theta), \\
\tau & =\sqrt{\varepsilon} T,
\end{aligned}
$$

and substituting equation (21) into equation (11), we have

$$
\begin{aligned}
& \frac{\partial I}{\partial \tau}=\frac{\mu}{2}\left(\omega^{2}+\sqrt{\varepsilon} I\right)-\alpha \sqrt{\omega^{2}+\sqrt{\varepsilon} I} \cos \theta, \\
& \frac{\partial \theta}{\partial \tau}=-\beta I+\sqrt{\varepsilon} \alpha \frac{\sin \theta}{\sqrt{\omega^{2}+\sqrt{\varepsilon} I}},
\end{aligned}
$$

with a Hamilton energy

$$
E(I, \theta)=\frac{\beta}{2} I^{2}+\frac{\mu}{2} \omega^{2} \theta-\omega \alpha \sin \theta .
$$




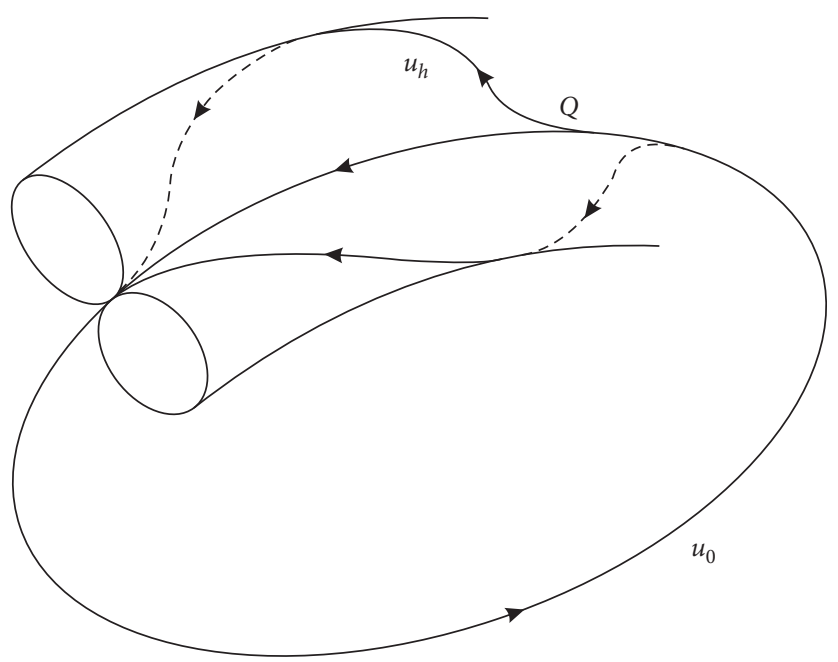

FIgURE 1: Schematic diagram of the homoclinic tubes.

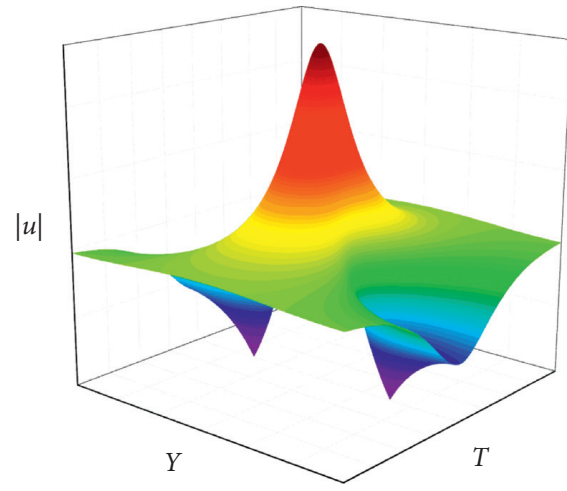

(a)

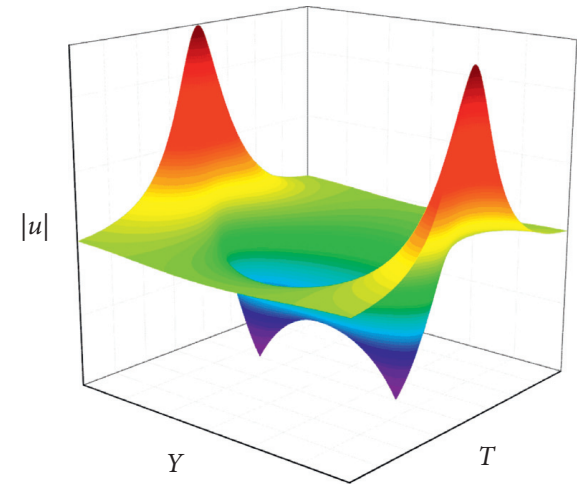

(b)

Figure 2: The exact form of the heteroclinic orbit for equation (17).

3.1. First Measurement. Note that the first measurement, the Melnikov method [22-25], shown in Figure 3(a), can be expressed as

$$
\begin{aligned}
\Delta & =\operatorname{Re} \int_{-\infty}^{+\infty} \int_{0}^{1} i\left(\frac{\mu}{2} \frac{\partial p_{h}}{\partial T} p_{h}-\alpha \frac{\partial p_{h}}{\partial T}\right) \mathrm{d} X \mathrm{~d} T \\
& =2 \omega \sqrt{2 \beta-1}\left(\frac{\mu}{2} \phi \omega^{3}\left(1-\frac{2}{3} \phi^{2}\right)+\alpha \sin 2 \phi \cos 2 \theta\right) .
\end{aligned}
$$

To ensure the transversality of $\mathrm{SM} W_{\varepsilon}^{s}(\Pi)$ and USM $W^{u}\left(Q_{\varepsilon}\right)$ holds, we have

$$
\frac{\mu}{2} \phi \omega^{3}\left(1-\frac{2}{3} \phi^{2}\right)+\alpha \sin 2 \phi \cos 2 \theta=0 .
$$

Thus, the persistence of the heteroclinic structure in $W^{u}\left(Q_{\varepsilon}\right) \cup W_{\varepsilon}^{s}(\Pi)$ is confirmed.

3.2. Second Measurement. According to [27-30], the second measurement, called as the geometric analysis (Figure 3(b)), is described as

$$
\frac{\mu}{2} \phi \omega^{3}+\alpha \sin 2 \phi \cos 2 \theta=0
$$

Inserting equation (23) into equation (24) and using $|\cos 2 \theta| \leq 1$, the chaotic threshold can be derived as

$$
\frac{\alpha}{\mu} \leq \frac{1}{2 \omega^{2}}\left|\frac{3 \sin \left(2 \arctan \sqrt{2 \beta \omega^{2}-\omega^{2}}\right)}{\omega \arctan \sqrt{2 \beta \omega^{2}-\omega^{2}}\left(3-2 \arctan ^{2} \sqrt{2 \beta \omega^{2}-\omega^{2}}\right)}\right|,
$$

where $\beta>(1 / 2)$. The chaotic threshold curves, i.e., black, red, and blue curves, denote the threshold curve (25) as $\beta=1.0$, 2.0, and 3.0 in Figure 4, respectively. The chaotic motions of the GBE may be generated as the ratio $\alpha / \mu$ locates below the curve.

\section{Numerical Simulation}

To test the aforementioned chaotic threshold (25), the differential quadrature method [27-29] in MATLAB is employed using equation (1) to study the influence of the damping coefficient $\mu$ on the nonlinear dynamical behaviors 


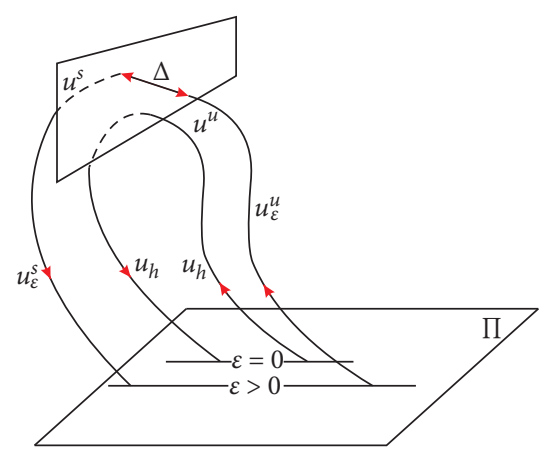

(a)

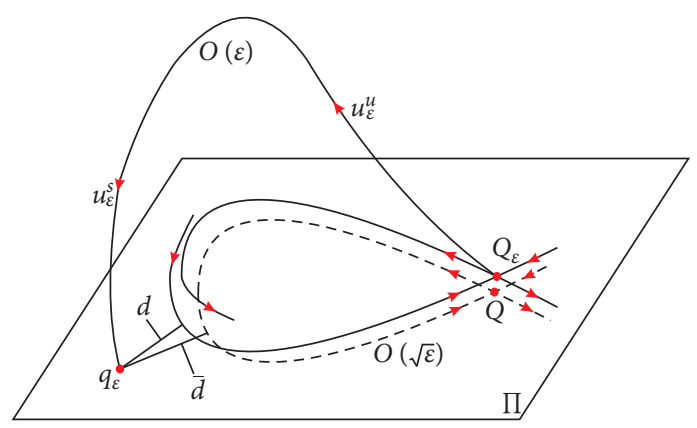

(b)

Figure 3: (a) The first measurement. (b) The second measurement.

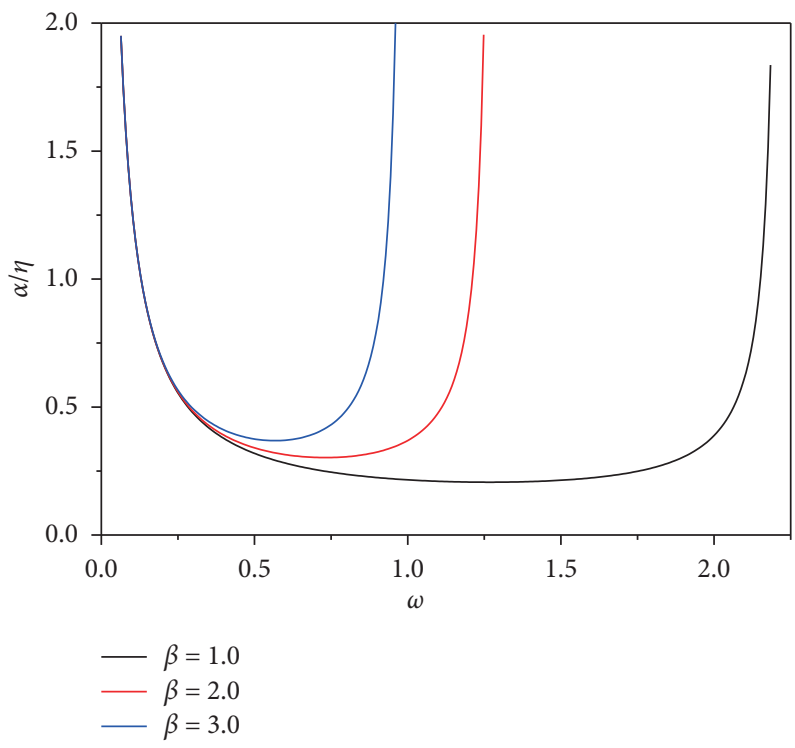

FIgURE 4: The chaotic threshold curve is plotted with the parameter ratio $\alpha / \mu$ via the parameter $\omega$.

of the GBE with cubic nonlinearity. Letting $\alpha=1.5$ and $\omega=1.0$, Figure 5 displays the bifurcation diagram, where the abscissa and ordinate represent the damping coefficient $\mu \in[0,1]$ and displacement of the GBE, respectively. The bifurcation behaviors show that the increase of damping coefficient can enhance the complicated dynamics of the GBE in comparison to the results in [27].

The chaotic wave in space $(x, t, q)$ is presented in Figure 6 as damping coefficient $\mu=0.3$. Figure 7 illustrates the chaotic motion for the GBE when damping coefficient $\mu=0.3$. Figure 7 (a) shows the phase trajectory on the plane $\left(q_{7}, \dot{q}_{7}\right)$, Figure $7(\mathrm{~b})$ presents the time response on the plane $\left(t, q_{7}\right)$, Figure $7(\mathrm{c})$ is the phase trajectory on the plane $\left(q_{13}, \dot{q}_{13}\right)$, Figure $7(\mathrm{~d})$ denotes the time response on the plane $\left(t, q_{13}\right)$, and Figures $7(\mathrm{e})$ and $7(\mathrm{f})$ are the phase trajectories in 3D spaces $\left(q_{7}, \dot{q}_{7}, q_{13}\right)$ and $\left(q_{13}, \dot{q}_{13}, q_{7}\right)$, respectively.

The chaotic behavior in $3 \mathrm{D}$ space $(x, t, q)$ as $\mu=0.63$ is presented in Figure 8. Figure 9 displays the chaotic phenomenon when the damping coefficient $\mu=0.63$. When $\mu$ is increased to 0.71 , Figures 10 and 11 illustrate the chaotic motion and chaotic wave for the GBE, respectively. When the damping coefficient $\mu=0.80$, the chaotic motion and chaotic wave are depicted, respectively, in Figures 12 and 13. The observed results from Figures 6 to 13 show that the amplitude of the GBE is larger for smaller $\mu$. However, the higher peak value of the truss core sandwich plate system is exhibited when the damping coefficient is sufficiently small or large [27]. 


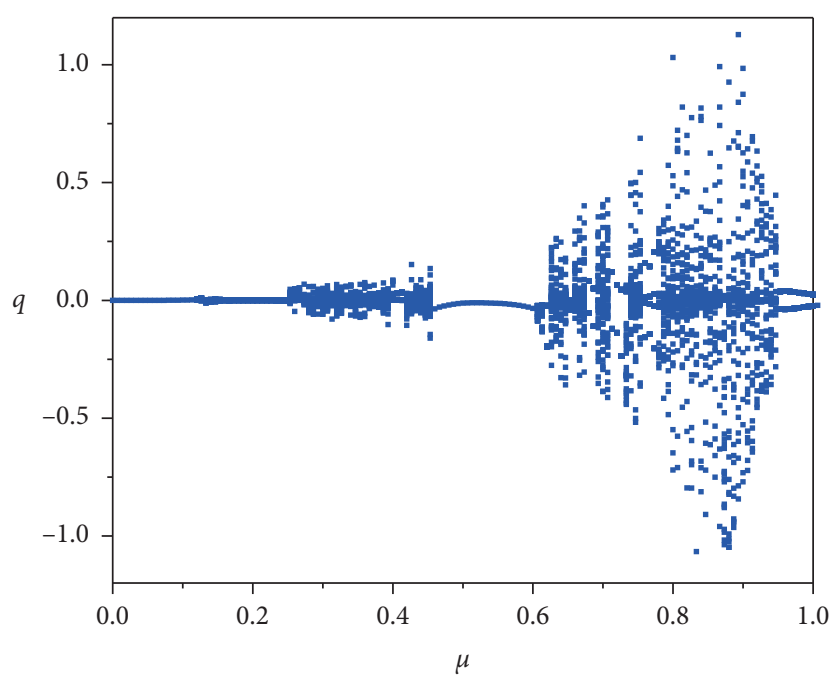

FIgURE 5: The bifurcation diagram of the composite laminated plate is plotted as $q$ via the damping coefficient $\mu$.

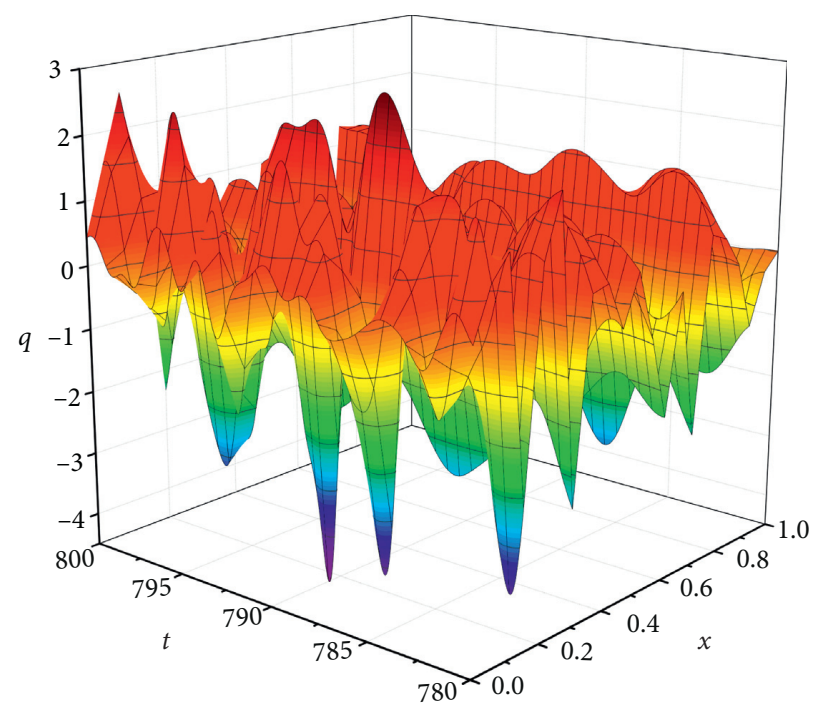

FIgURE 6: The chaotic wave in space $(x, t, q)$ when damping coefficient $\mu=0.3$.

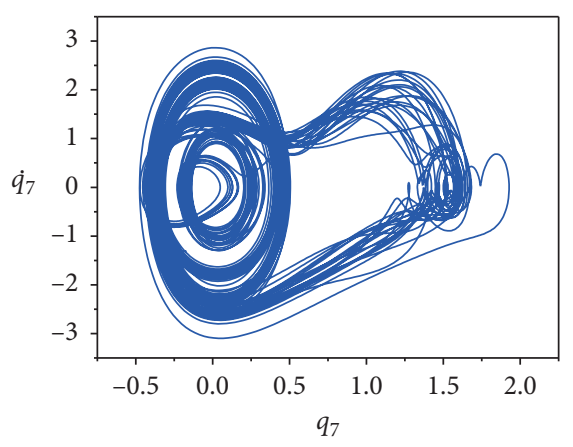

(a)

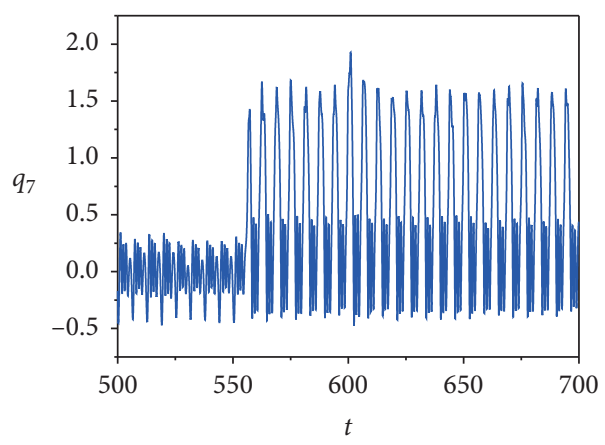

(b)

FIgURE 7: Continued. 


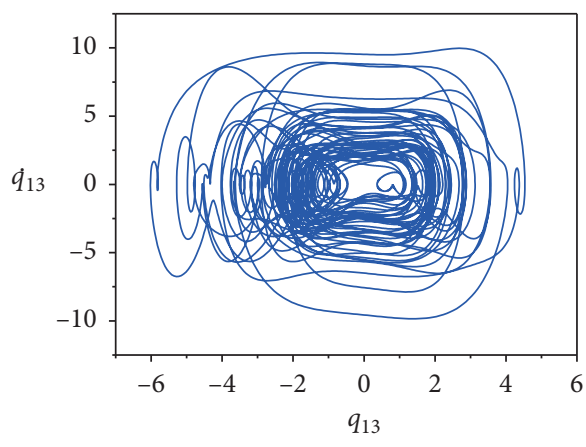

(c)

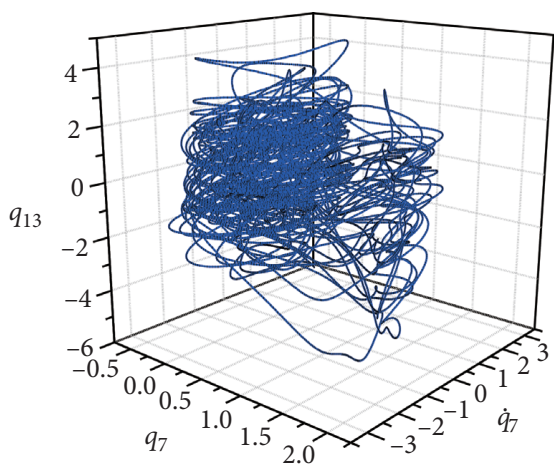

(e)

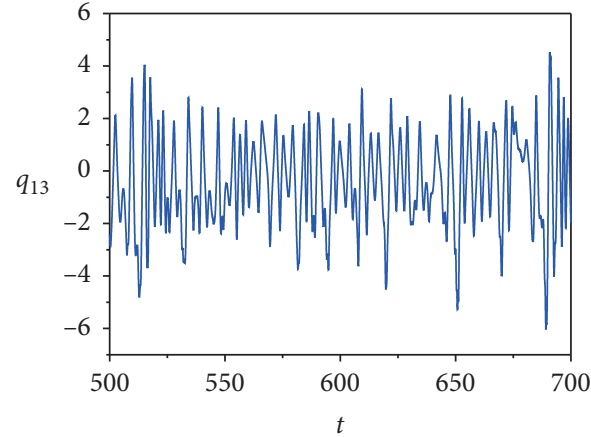

(d)

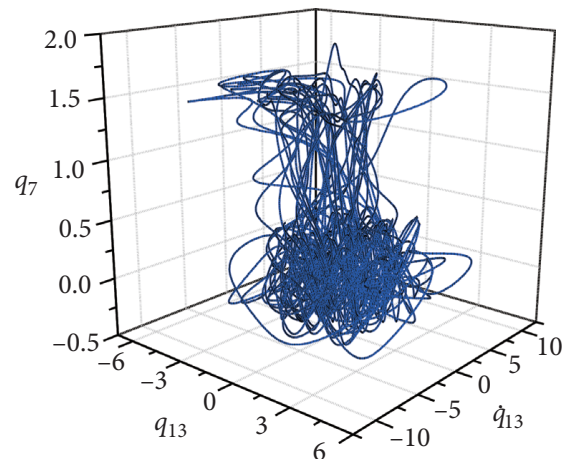

(f)

Figure 7: The chaotic motion when damping coefficient $\mu=0.3$. (a) The phase trajectory on plane $\left(q_{7}, \dot{q}_{7}\right)$. (b) The phase trajectory on plane $\left(q_{13}, \dot{q}_{13}\right)$. (c) The time response on plane $\left(t, q_{7}\right)$. (d) The time response on plane $\left(t, q_{13}\right)$. (e) The phase trajectory in $3 \mathrm{D}$ space $\left(q_{7}, \dot{q}_{7}, q_{13}\right)$. (f) The phase trajectory in 3D space $\left(q_{13}, \dot{q}_{13}, q_{7}\right)$.

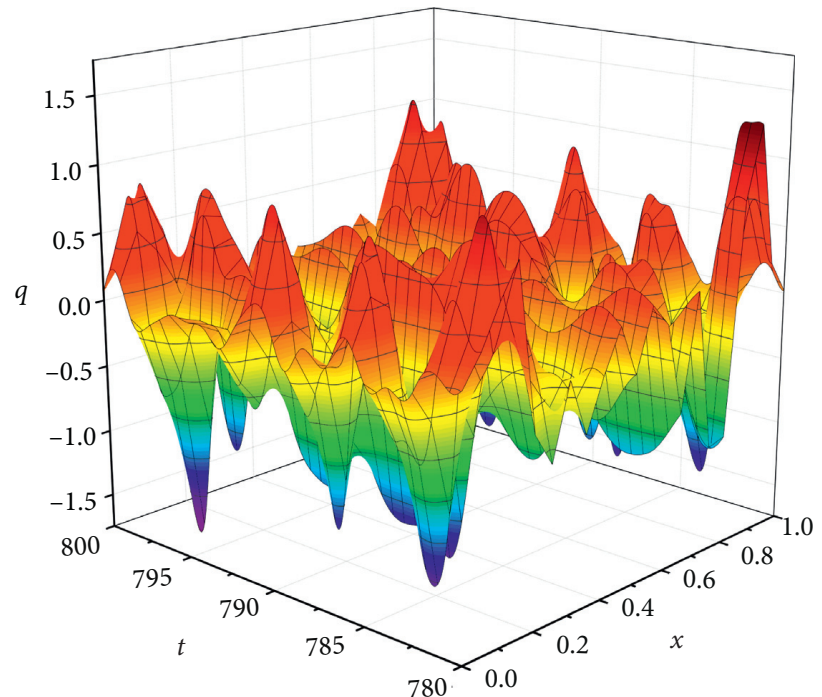

Figure 8: The chaotic wave in space $(x, t, q)$ when damping coefficient $\mu=0.63$. 


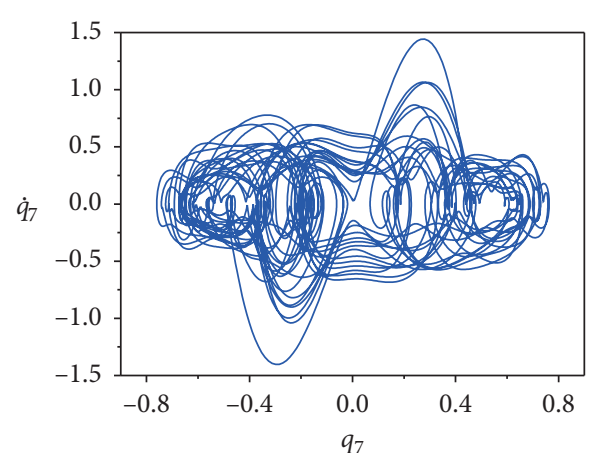

(a)

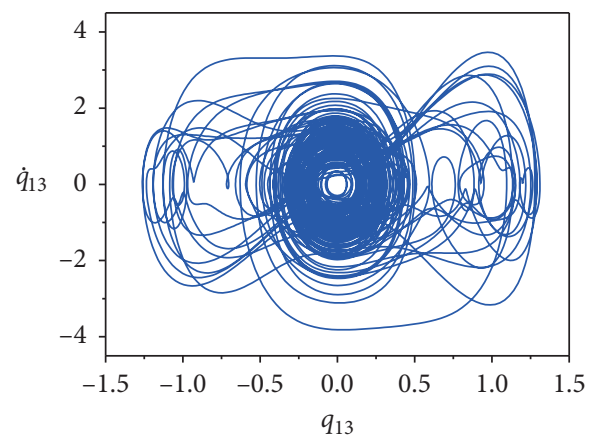

(c)

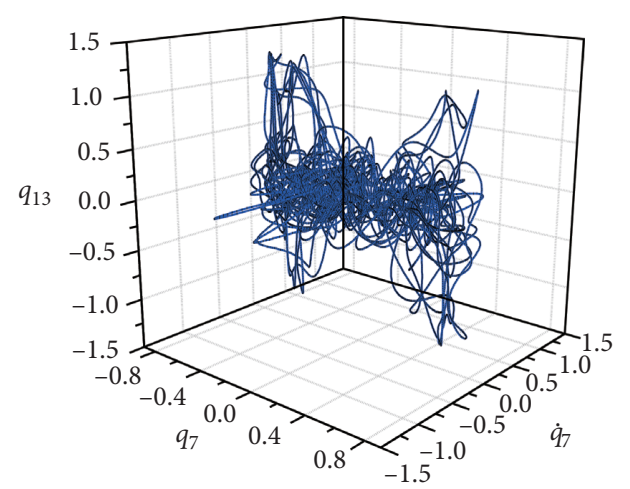

(e)

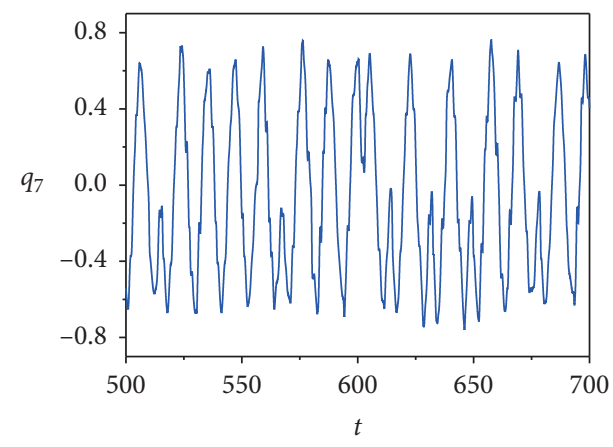

(b)

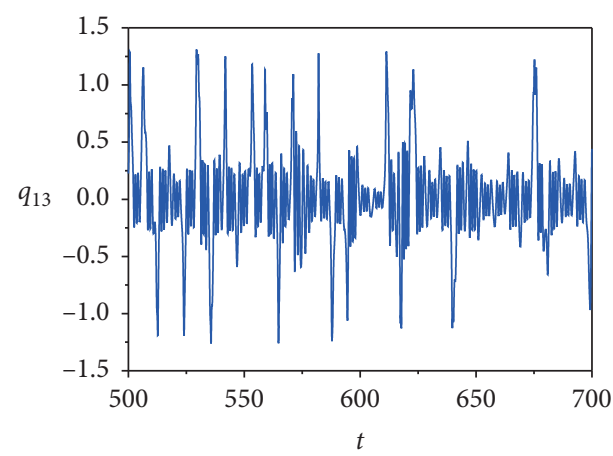

(d)

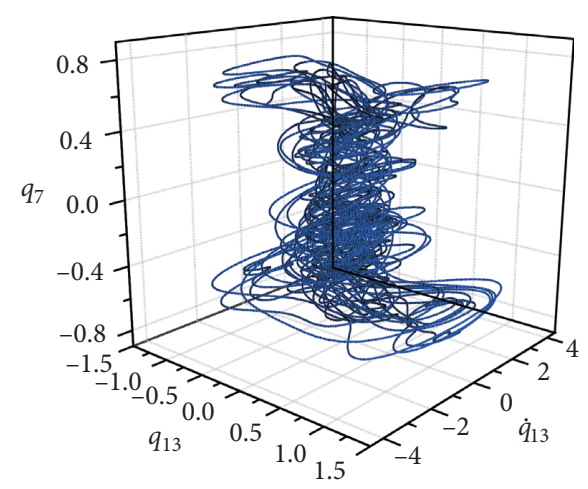

(f)

Figure 9: The chaotic motion when damping coefficient $\mu=0.63$.

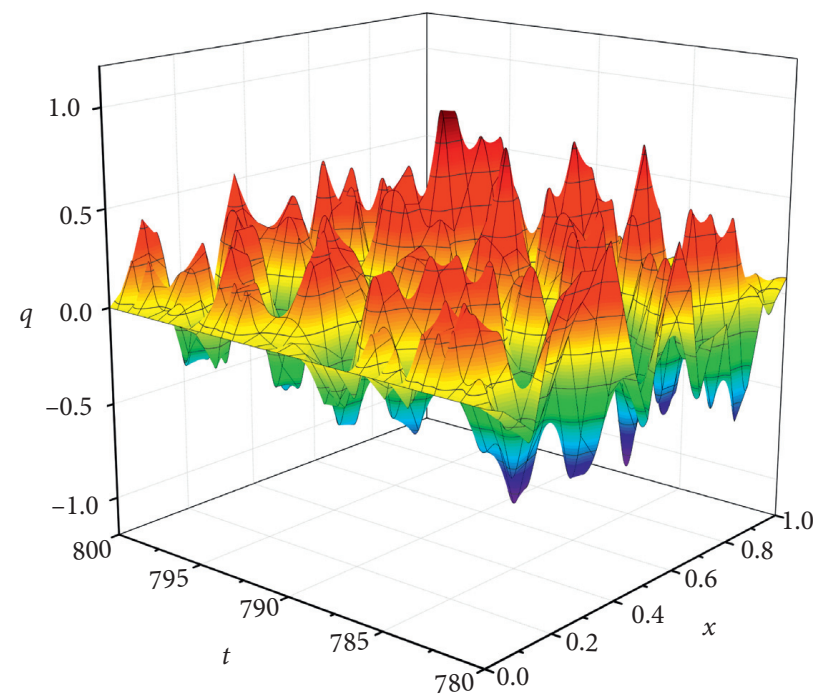

Figure 10: The chaotic wave in space $(x, t, q)$ when damping coefficient $\mu=0.71$. 


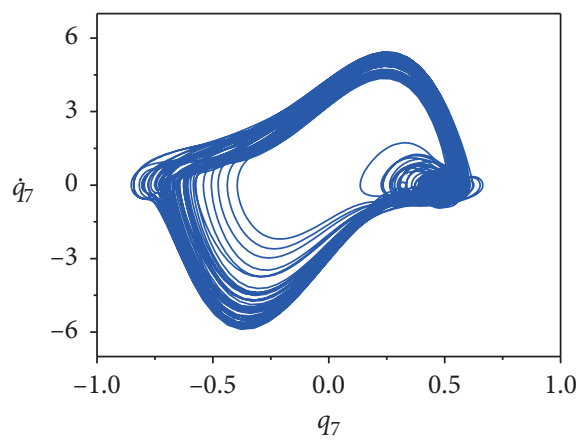

(a)

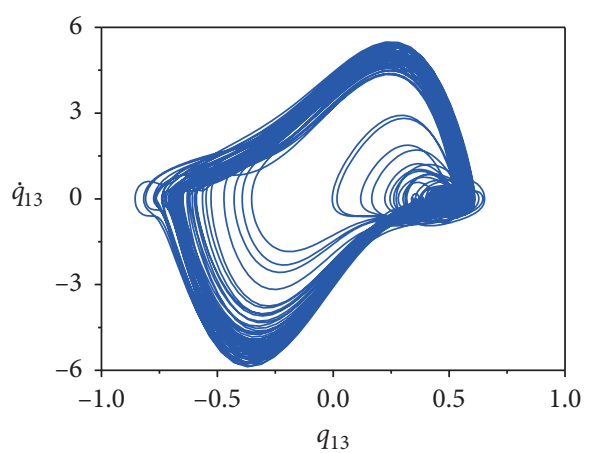

(c)

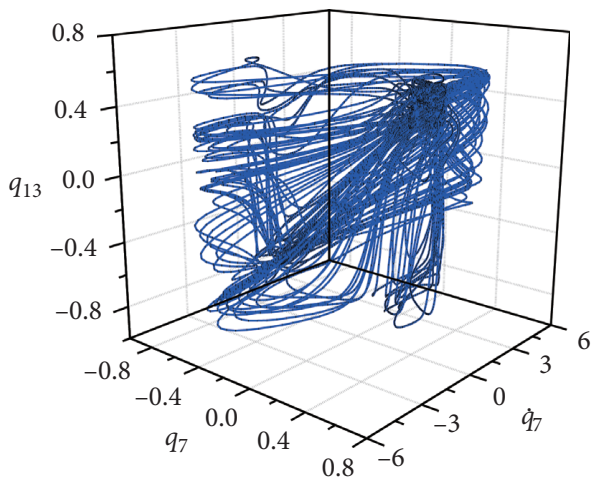

(e)

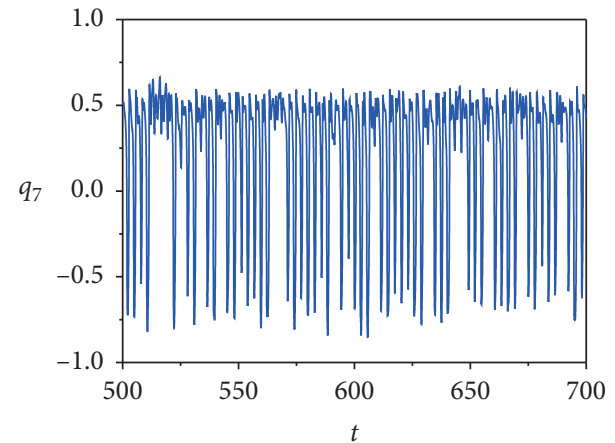

(b)

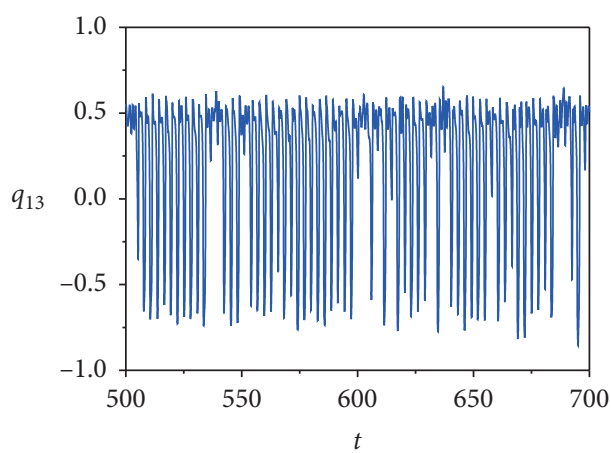

(d)

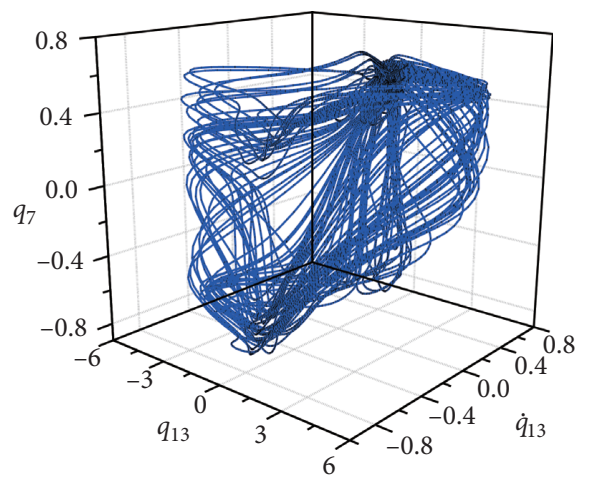

(f)

FIgURE 11: The chaotic motion when damping coefficient $\mu=0.71$.

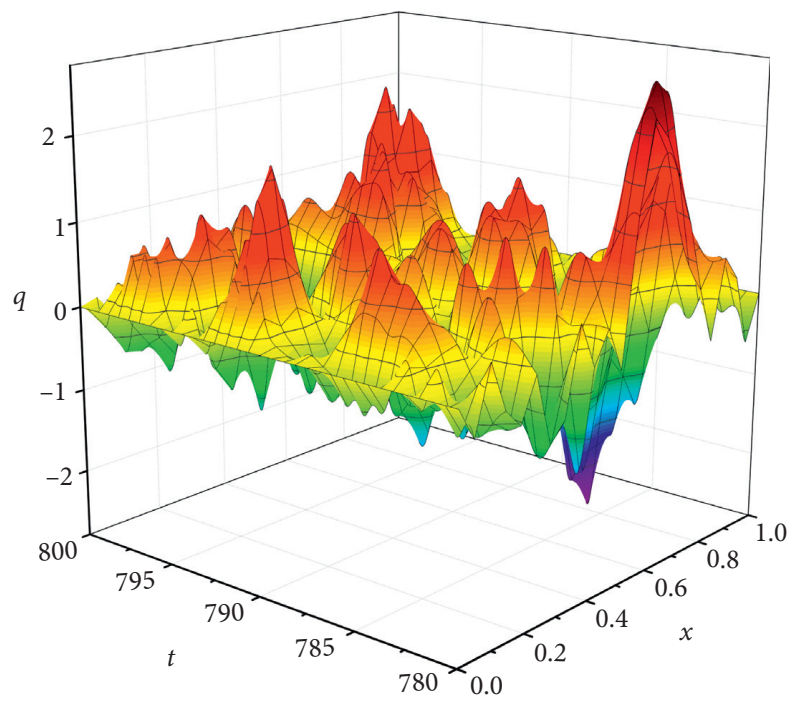

FIGURE 12: The chaotic wave in space $(x, t, q)$ when damping coefficient $\mu=0.80$. 


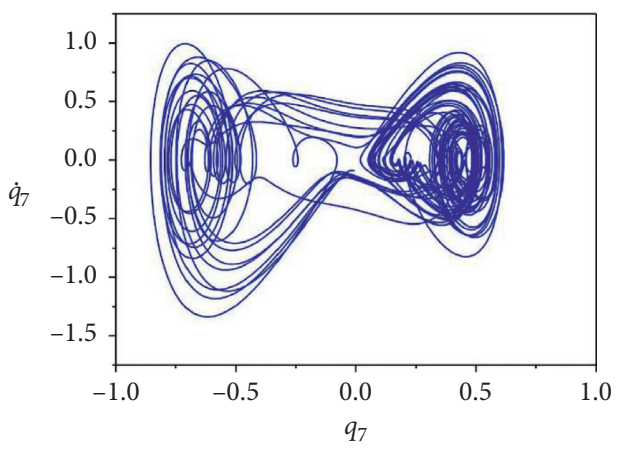

(a)

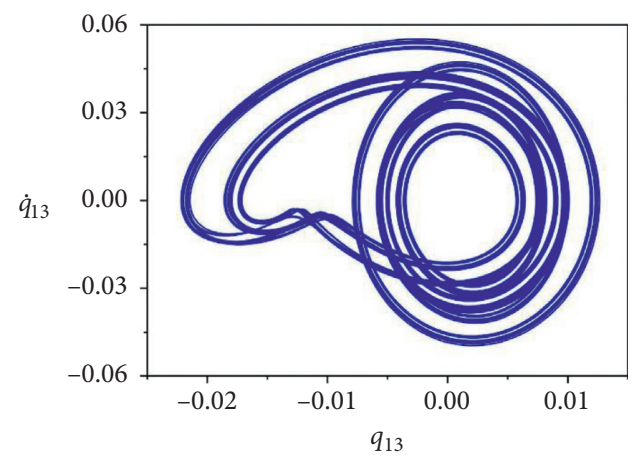

(c)

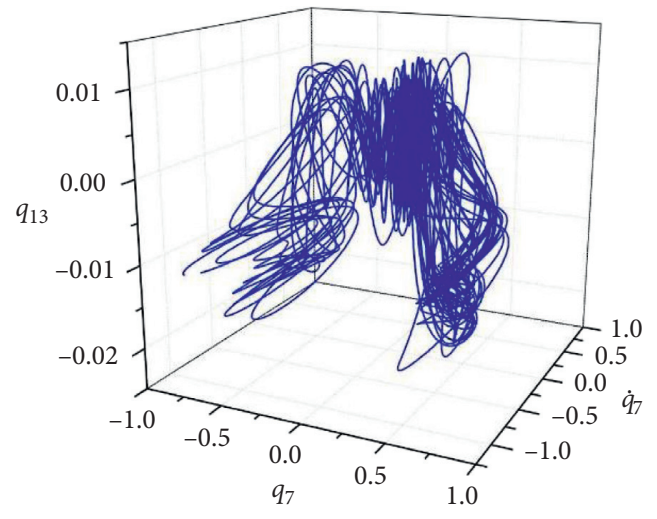

(e)

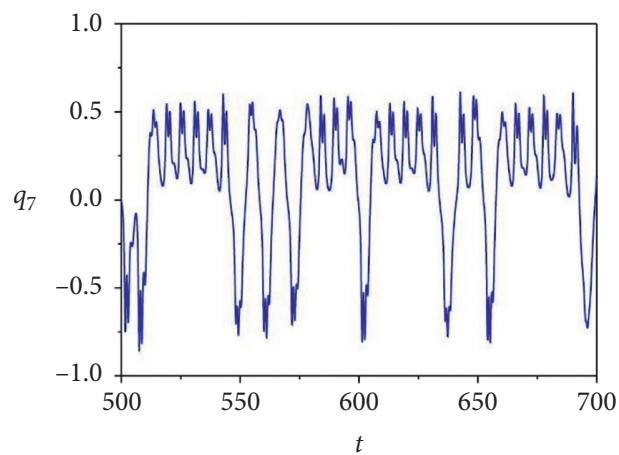

(b)

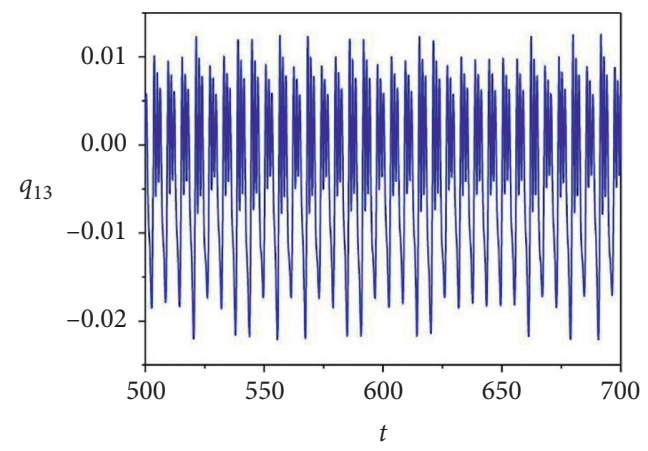

(d)

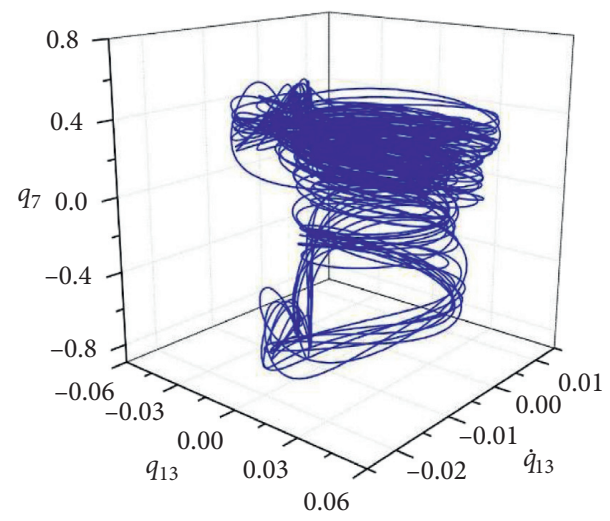

(f)

FIgURE 13: The chaotic motion when damping coefficient $\mu=0.80$.

\section{Conclusions}

The global perturbation method for the infinite-dimensional system was used to discuss global dynamics for the GBE in this work. Using the RPM, an analytical wave equation was obtained by simplifying the complicated nonlinear equation of the GBE. Afterward, the persistent homoclinic structure for wave equations was constructed by using the first and second measurements. Finally, quantitative analysis was conducted to examine the theoretical results using the differential quadrature method.
The numerical studies illustrate that the damping coefficient $\mu$ is essential to determine the dynamical responses of the GBE. Different bifurcation phenomena are shown with changing damping coefficient $\mu$. Therefore, nonlinear wave responses for the GBE can be controlled by adjusting the damping coefficient.

\section{Data Availability}

The data used to support the findings of this study are included within the article. 


\section{Conflicts of Interest}

The authors declare that they have no conflicts of interest.

\section{Acknowledgments}

The authors thank the National Natural Science Foundation of China (11902220, 11290152, and 11427801) and the Funding Project for Academic Human Resources Development in Institutions of Higher Learning under the Jurisdiction of Beijing Municipality for supporting this study.

\section{References}

[1] V. Roeber and J. D. Bricker, "Destructive tsunami-like wave generated by surf beat over a coral reef during Typhoon Haiyan," Nature Communications, vol. 6, p. 7854, 2015.

[2] T. Shimozono, Y. Tajima, A. B. Kennedy, H. Nobuoka, J. Sasaki, and S. Sato, "Combined infragravity wave and seaswell runup over fringing reefs by super typhoon Haiyan," Journal of Geophysical Research: Oceans, vol. 120, no. 6, pp. 4463-4486, 2015.

[3] V. Roeber and K. F. Cheung, "Boussinesq-type model for energetic breaking waves in fringing reef environments," Coastal Engineering, vol. 70, pp. 1-20, 2012.

[4] J. Weiss, M. Tabor, and G. Carnevale, “The Painlevé property for partial differential equations," Journal of Mathematical Physics, vol. 24, no. 3, pp. 522-526, 1983.

[5] J. Weiss, "The Painlevé property for partial differential equations. II: Bäcklund transformation, Lax pairs, and the Schwarzian derivative," Journal of Mathematical Physics, vol. 24, no. 6, pp. 1405-1413, 1983.

[6] J. Weiss, "The Painlevé property and Bäcklund transformations for the sequence of Boussinesq equations," Journal of Mathematical Physics, vol. 26, no. 2, pp. 258-269, 1985.

[7] K. Mohammed, "Exact traveling wave solutions of the Boussinesq-Burgers equation," Mathematical and Computer Modelling, vol. 49, pp. 66-671, 2009.

[8] X. Yang, C. Zhao, and J. Cao, "Dynamics of the discrete coupled nonlinear Schrödinger-Boussinesq equations," Applied Mathematics and Computation, vol. 219, no. 16, pp. 8508-8524, 2013.

[9] M. A. Helal, A. R. Seadawy, and M. H. Zekry, "Stability analysis of solitary wave solutions for the fourth-order nonlinear Boussinesq water wave equation," Applied Mathematics and Computation, vol. 232, pp. 1094-1103, 2014.

[10] M. Awais and S. Ibrahim, "Nonlinear instability for the Boussinesq equations with diabatic forcing," Nonlinear Analysis, vol. 166, pp. 1-18, 2018.

[11] Z. Yang, J. Tang, and Y. Shen, "Numerical study for vegetation effects on coastal wave propagation by using nonlinear Boussinesq model," Applied Ocean Research, vol. 70, pp. 3240, 2018.

[12] J. Cai, J. Chen, and B. Yang, "Efficient energy-preserving wavelet collocation schemes for the coupled nonlinear Schrödinger-Boussinesq system," Applied Mathematics and Computation, vol. 357, pp. 1-11, 2019.

[13] S. Wang and X. Su, "The Cauchy problem for the dissipative Boussinesq equation," Nonlinear Analysis: Real World Applications, vol. 45, pp. 116-141, 2019.

[14] K. Yildirim, "On the boundary control of a Boussinesq system," Journal of Informatics and Mathematical Sciences, vol. 12, pp. 199-208, 2020.
[15] K. Yildirim, "Active control of an improved Boussinesq system," Mathematical Modelling of Natural Phenomena, vol. 15, p. 58, 2020.

[16] R. Tian, Q. Cao, and S. Yang, "The codimension-two bifurcation for the recent proposed SD oscillator," Nonlinear Dynamics, vol. 59, no. 1-2, pp. 19-27, 2010.

[17] Q. L. Wu, W. Zhang, and E. H. Dowell, "Detecting multi-pulse chaotic dynamics of high-dimensional non-autonomous nonlinear system for circular mesh antenna," International Journal of Non-linear Mechanics, vol. 102, pp. 25-40, 2018.

[18] Q. L. Wu and G. Y. Qi, "Viscoelastic string-beam coupled vibro-impact system: modeling and dynamic analysis," European Journal of Mechanics A-Solid, vol. 82, Article ID $104012,2020$.

[19] R. L. Tian, Z. J. Zhao, and Y. Xu, "Variable scale-convex-peak method for weak signal detection," Science China Technological Sciences, vol. 63, 2020.

[20] Q. Wu and G. Qi, "Quantum dynamics for Al-doped graphene composite sheet under hydrogen atom impact," Applied Mathematical Modelling, vol. 90, pp. 1120-1129, 2021.

[21] Q. Wu, M. Yao, M. Li, D. Cao, and B. Bai, "Nonlinear coupling vibrations of graphene composite laminated sheets impacted by particles," Applied Mathematical Modelling, vol. 93, pp. 75-88, 2021.

[22] Y. Niu, W. Zhang, and X. Y. Guo, "Free vibration of rotating pretwisted functionally graded composite cylindrical panel reinforced with graphene platelets," European Journal of Mechanics- A Solids, vol. 77, Article ID 103798, 2019.

[23] Y. Li and D. W. McLaughlin, "Homoclinic orbits and chaos in discretized perturbed NLS systems: Part I. Homoclinic orbits," Journal of Nonlinear Science, vol. 7, no. 3, pp. 211-269, 1997.

[24] Y. Li and S. Wiggins, "Homoclinic orbits and chaos in discretized perturbed NLS systems: Part II. Symbolic dynamics," Journal of Nonlinear Science, vol. 7, no. 4, pp. 315-370, 1997.

[25] C. Zeng, "Homoclinic orbits for a perturbed nonlinear Schrödinger equation," Communications on Pure and Applied Mathematics, vol. 53, no. 10, pp. 1222-1283, 2000.

[26] J. Shatah and C. Zeng, "Homoclinic orbits for the perturbed Sine-Gordon equation," Communications on Pure and Applied Mathematics, vol. 53, no. 3, pp. 283-299, 2000.

[27] W. Zhang, Q. L. Wu, M. H. Yao, and E. H. Dowell, “Analysis on global and chaotic dynamics of nonlinear wave equations for truss core sandwich plate," Nonlinear Dynamics, vol. 94, no. 1, pp. 21-37, 2018.

[28] Q. Wu and G. Qi, "Global dynamics of a pipe conveying pulsating fluid in primary parametrical resonance: analytical and numerical results from the nonlinear wave equation," Physics Letters A, vol. 383, no. 14, pp. 1555-1562, 2019.

[29] Q. Wu and G. Qi, "Homoclinic bifurcations and chaotic dynamics of non-planar waves in axially moving beam subjected to thermal load," Applied Mathematical Modelling, vol. 83, pp. 674-682, 2020.

[30] M. Li, Q. Wu, and B. Bai, "Size-dependent mechanics of viscoelastic carbon nanotubes: modeling, theoretical and numerical analysis," Results in Physics, vol. 19, Article ID 103383, 2020.

[31] J. Boussinesq, "Théorie des ondes et des remous qui se propagent le long d'un canal rectangulaire horizontal," Journal de mathématiques pures et appliquées, vol. 2, pp. 55-108, 1872. 